\title{
Skeletal metastases in advanced pancreatic ductal adenocarcinoma: a retrospective analysis
}

\author{
Akshjot Puri ${ }^{1}$, John Chang ${ }^{2}$, Natalee Tanner ${ }^{3}$, Patricia Lucente ${ }^{3}$, Janice Desiongco ${ }^{3}$, Tomislav Dragovich ${ }^{4}$, \\ Boris Naraev ${ }^{4}$, Madappa Kundranda ${ }^{4}$ \\ ${ }^{1}$ Department of Hematology/Oncology, Houston Methodist, Houston, TX, USA; ${ }^{2}$ Department of Radiology, Banner MD Anderson Cancer Center, \\ Gilbert, AZ, USA; ${ }^{3}$ Banner MD Anderson Cancer Center, Gilbert, AZ, USA; ${ }^{4}$ Division of Cancer Medicine, Banner MD Anderson Cancer Center, \\ Gilbert, AZ, USA \\ Contributions: (I) Conception and design: M Kundranda, J Chang, A Puri; (II) Administrative support: M Kundranda, T Dragovich, B Naraev, N \\ Tanner, P Lucente, J Desiongco; (III) Provision of study materials or patients: M Kundranda, T Dragovich, B Naraev, J Chang, N Tanner, P Lucente, \\ J Desiongco; (IV) Collection and assembly of data: A Puri; (V) Data analysis and interpretation: A Puri, M Kundranda, B Naraev; (VI) Manuscript \\ writing: All authors; (VII) Final approval of manuscript: All authors. \\ Correspondence to: Akshjot Puri, MD. Clinical Fellow, Department of Hematology/Oncology, Houston Methodist, 6445 Main St., Houston, TX \\ 77030, USA. Email: apuri@houstonmethodist.org.
}

Background: Skeletal metastases (SM) in advanced pancreatic ductal adenocarcinoma (PDAC) is an infrequent occurrence that has been previously reported in literature to occur in less than $2.5 \%$ of the cases. Complications such as pathological fractures can result in intractable pain, immobilization and a significant deterioration in quality of life. The purpose of this study is to improve the understanding of the increasing incidence of SM and the importance of surveillance and adequate management of SM in these patients.

Methods: A retrospective analysis was conducted using a clinical database at a single tertiary care institution for cancer patients; this included 207 patients with advanced PDAC diagnosed between December 2004 and March 2017 receiving palliative chemotherapy. SM were identified by computerized tomography (CT)/ fluorodeoxyglucose positron emission tomography (FDG-PET)/magnetic resonance imaging (MRI). Information regarding demographics, clinical course and date of last follow-up/death were collected. After a median follow-up of 11 months, an analysis was conducted, including a Kaplan-Meier survival analysis.

Results: The study included 207 patients; 19 out of 207 patients (9.2\%) developed SM; the primary tumor was located in the pancreatic body/tail in 12 out of 19 patients (63.2\%). The thoracic and lumbar vertebrae were the most common sites of SM. Other common synchronous sites of metastases included the liver and lung. A majority of the lesions were osteolytic (63.2\%). The median time of diagnosis from the initial diagnosis was 2 months (range, 0-60 months). Bone pain was observed as the initial symptom in 7 out of 19 patients (36.8\%), 2 out of 19 patients (10.5\%) had a pathological fracture and 1 out of 19 patients (5.3\%) developed a para-spinal mass causing inferior vena cava compression. The median survival period for patients with SM was 11 months (range, 0-62 months) and for those without SM was 12 months (range, 0-147 months) [hazard ratio (HR) 1.24, 95\% confidence interval (CI): 0.66-2.30, $\mathrm{P}=0.51$ ].

Conclusions: There has been a challenge with regards to management of the increasing number of patients with SM. Thoracic and lumbar vertebrae are the most common sites and pathological fractures in these sites can be catastrophic. Careful evaluation of skeletal signs and symptoms, early detection and intervention are essential to prevent morbidity and mortality from complications in patients with PDAC and SM.

Keywords: Skeletal metastases (SM); advanced pancreatic adenocarcinoma; incidence; intervention; overall survival

Submitted Aug 28, 2020. Accepted for publication Jan 31, 2021.

doi: 10.21037/jgo-20-361

View this article at: http://dx.doi.org/10.21037/jgo-20-361 


\section{Introduction}

Pancreatic ductal adenocarcinoma (PDAC) is a malignancy where, while the overall incidence of the cancer is only $3 \%$ in the general population, the mortality rate closely approaches the incidence rates (1). The survival of PDAC patients declines rapidly with its spread; the estimated 5-year survival of patients with localized disease is $31.5 \%$, with regionally spread disease at $11.5 \%$ and with distantly spread at $2.7 \%$ (2). With the advent of newer combination systemic therapies like FOLFIRINOX and gemcitabine plus nabpaclitaxel, the 5 -year survival in advanced disease is now improved to about $8-10 \%$, as compared to 3\% in 1975 (2-8).

The skeletal system has been a known common site of metastases for various malignancies like prostate, breast, and lung cancers, and has also been associated with kidney, bladder, and thyroid cancers, as well as some lymphomas and sarcomas (9). Established data demonstrate that prostate cancer typically leads to osteoblastic lesions in the skeletal system, while other forms of cancers such as breast cancer usually cause osteolytic lesions (9). The most common sites of metastases in PDAC are the liver and peritoneal cavity whereas skeletal metastases (SM) from pancreatic cancer have thus far been considered an infrequent occurrence. The first case of pancreatic cancer with SM was described in 1963 (10). A study done in 2009, included 323 patients with advanced PDAC and reported an incidence of $2.2 \%$ of SM $(11,12)$. There is an observed increase in the incidence of SM in PDAC patients which perhaps is related to the increased overall survival with novel systemic treatment regimens. Complications such as pathological fractures in these patients can result in intractable pain, immobilization and a significant deterioration in quality of life.

The most common sites of SM from PDAC are reported to be in vertebrae, pelvis and ribs (12). The primary tumor is commonly located in the body/tail of the pancreas as seen in 10 out of 13 patients in a retrospective study (13), albeit the exact pathogenesis is unknown. Preliminary data suggest that cytokines such as interleukin 6 (IL-6), vascular endothelial growth factor (VEGF), and parathyroid hormone-related protein (PTHrP) may play a pivotal role in the growth of pancreatic cancer in the bone (13). Other factors that stimulate osteoclastic bone resorption such as transforming growth factor beta (TGF-beta), interleukin 11 (IL-11) and matrix metalloproteinases may be involved as well (14). It has been suggested that the pathogenesis of SM is probably related to the Batson's vertebral vein plexus, which bypasses the liver and lungs. This plexus is longitudinal, valveless and extends from the sacrum to the skull. Tumor cells can enter the Batson's vertebral vein plexus and spread to the vertebrae, ribs, pelvis and proximal limb girdle (9). This may explain why the cancer of the head of the pancreas very rarely causes SM as compared to the cancer of the body or the tail of the pancreas. In addition, bone involvement in patients with pancreatic cancer may result from direct posterior extension of the primary tumor with destruction of the bodies of one or more upper lumbar vertebrae $(11,15)$. Additional factors such as ethnic, genetic, and biologic variables that determine the homing and proliferation of tumor cells and incidence of SM in PDAC are still poorly understood.

The SM from PDAC are characterized as being predominantly osteoblastic in some (12) and osteolytic in other reports (13). There is very limited literature available to assess incidence, presentation, risk factors, clinical and demographic characteristics and time of occurrence with respect to PDAC diagnosis. Also, very little is known about its natural history, survival and overall prognosis of SM in PDAC. The most common presenting symptoms of SM from PDAC are non-specific, e.g., back pain. As per a report, a 77-year-ld man presented with back pain and was found to have osteoblastic changes of L3 vertebrae on imaging (10). Subsequent computed tomography (CT) of the abdomen demonstrated enlarged peri-pancreatic lymph nodes and fluid in the space of Douglas (11). Biopsy of the L3 lesion led to the initial diagnosis of a well-differentiated pancreatic adenocarcinoma (11). There is a case report of SM presenting as the only site of recurrence, three years after initial curative surgery and adjuvant gemcitabine in a patient with resectable PDAC (16). Another case reported a 25-year-old male who was found to have bilateral temporal bone adenocarcinoma caused by metastases from a primary lesion in the pancreas after he presented with hearing impairment, as the first site of metastases with newly diagnosed PDAC (17).

It is challenging to estimate the exact incidence of bone metastases from PDAC, since the imaging of the bones at initial diagnosis and examination of the skeleton at autopsy are not a part of standard of care for treatment for all patients. SM commonly leads to pathological fractures, uncontrolled pain, immobilization, impaired quality of life and emotional distress. In addition, complications like hypercalcemia and cord compression can become catastrophic. This can be prevented by early recognition and intervention such as palliative radiation, stabilization surgery and/or bone directed therapy. Thus, it is essential 
to address these gaps in our knowledge with respect to SM secondary to PDAC, which remain underreported, especially in the light of its increased occurrence.

\section{Objectives}

In this retrospective study, the primary objective was to describe the clinical characteristics of patients with advanced PDAC who developed SM. The secondary objectives of the study were to (I) describe SM related complications in advanced PDAC, (II) study association of worsening performance status (PS) with development of SM and (III) study association of survival between patients with and without SM in advanced PDAC.

We present the following article in accordance with STROBE reporting checklist (available at http://dx.doi. org/10.21037/jgo-20-361).

\section{Methods}

\section{Subjects and setting}

This study was a single institution observational study conducted at Banner MD Anderson Cancer Center in Gilbert, Arizona specializing in cancer patients. A retrospective analysis was performed on patients with advanced PDAC receiving palliative chemotherapy. The overall cohort consisted of patients aged 53 to 92 years with initial presentation of advanced pancreatic cancer between December, 2004 and March, 2017. During this time, patients underwent treatment for their pancreatic cancer as per the physician's choice. Only patients with available information regarding demographics, cancer staging, clinical course and follow-up were included. Patients with pancreatic neuroendocrine tumors were excluded. To eliminate selection bias, the study aimed at including all newly diagnosed advanced pancreatic cancers during the specified time frame in order to achieve a representative cross-section of this study population.

\section{Measures and outcomes}

Clinical and pathological data were extracted from the electronic medical record, which included patient demographics, Eastern Cooperative Oncology Group (ECOG) PS, pancreatic cancer stage, sites of primary and visceral metastatic disease as well as the chemotherapy regimens received by the patients. The study identified the number of patients who developed SM secondary to pancreatic cancer defined by imaging evidence of metastatic disease by computerized tomography (CT)/ fluorodeoxyglucose positron emission tomography (FDG$\mathrm{PET}) / \mathrm{magnetic}$ resonance imaging (MRI). Predictor variables such as time of onset of SM with respect to initial diagnosis of advanced pancreatic cancer, clinical characteristics like sites of skeletal metastatic lesions and pathological characteristics such as osteolytic/osteoblastic SM were collected. The study included information regarding complications such as pain and pathological fracture secondary to SM as well as dates of death or last follow-up. Confounding variables like ECOG PS, grade of toxicity from chemotherapy as per Common Terminology Criteria for Adverse Events (CTCAE) grading and tumor burden in terms of other sites of visceral metastatic disease sites were also collected.

\section{Follow-up}

The patients were followed in the gastrointestinal oncology outpatient clinic at the cancer center. The patients had a median follow-up of 11 months (range, 0.1-146 months) and the data was locked in January, 2018 when the analysis was performed. The data was collected by a single individual in a comparable manner to minimize measurement error. Patient confidentiality was maintained by de-identification, data storage in a password protected server, and access restricted only to the study investigators.

\section{Statistical analysis}

As determined by the study period, a fixed available sample size was used. Characteristics of the whole cohort and patients with SM were presented as median and interquartile ranges for continuous variables. Associations of ECOG PS with SM incidence was performed, and Fisher's exact test was used. Survival analysis was performed using Kaplan-Meier method and log-rank test was used to test the overall survival between the two groups. Patients who were alive were censored at the time of last follow-up for survival. Patients who had missing information due to no followup were excluded from the study and analysis. Univariable Cox proportional hazards model was used to assess OS by SM status. Statistical significance was defined as two tailed $\mathrm{P}<0.05$ for all tests. 

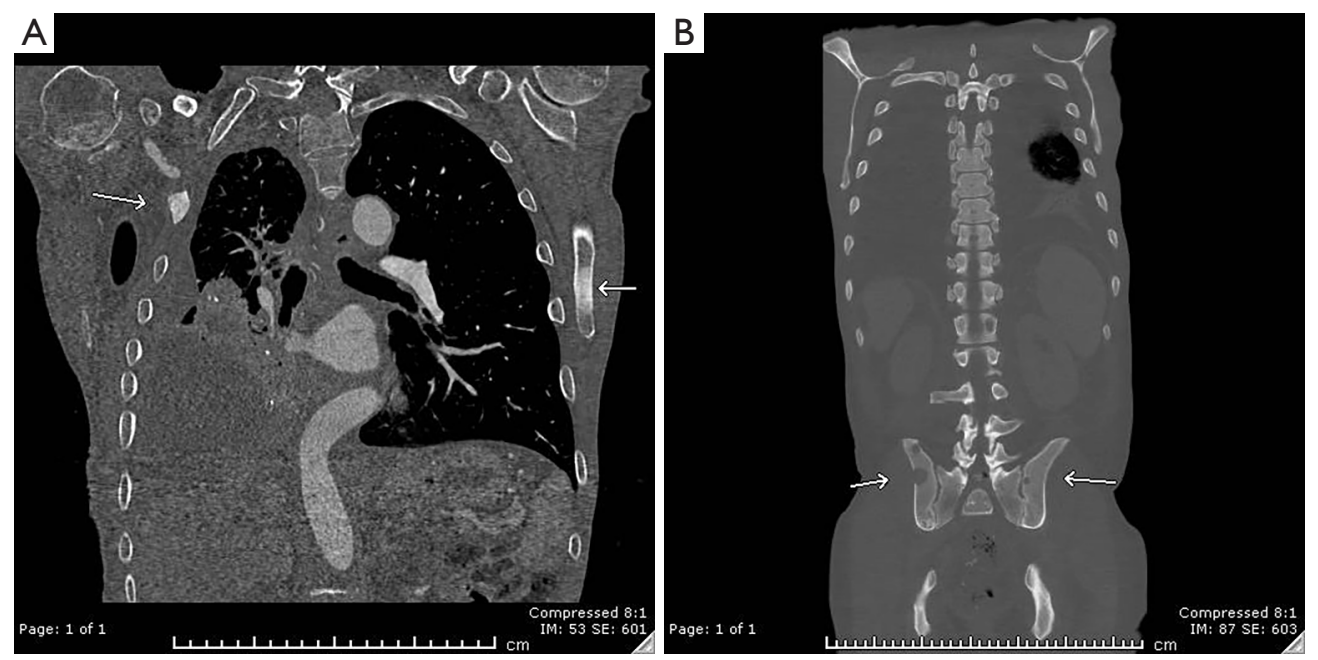

Figure 1 CT scan in bone window. (A) Coronal chest section with osteoblastic skeletal metastases (arrows); (B) coronal abdomen \& pelvis section with osteolytic skeletal metastases (arrows). CT, computed tomography.

\section{Ethical statement}

The study was conducted in accordance with the Declaration of Helsinki (as revised in 2013). The study was approved by the Institutional Review Board at Banner MD Anderson Cancer Center. (Project \# 14-15-0094) and individual consent for this retrospective analysis was waived.

\section{Results}

There were a total of 207 patients diagnosed with advanced stage PDAC, planned to receive palliative chemotherapy. These patients were followed in the outpatient clinic and patients with sufficient information for all data points were included in the study. The median age for the overall cohort was 66 years at the time of initial PDAC diagnosis (range, 53-92 years). The study included an equal distribution of males-103 out of 207 (49.8\%) and females-104 out of $207(50.2 \%)$. The primary tumor was located in the head/ neck of the pancreas in 124 patients (59.9\%), body/tail in 78 patients $(37.7 \%)$ and had an occult primary in 5 patients (2.4\%). About 74 out of 207 patients (35.7\%) had metastatic cancer at initial diagnosis. The median number of other sites of metastatic lesions was 1 (range, $0-4$ ) in the whole cohort with the most common sites being liver (24.6\%), lung $(7.7 \%)$, and peritoneum (7.2\%). The median number of lines of treatment was 1 (range, 0-6) for the whole cohort. There were 50 patients who received gemcitabine only, 113 patients received multiple lines of therapy, 42 patients were unable to get chemotherapy or received palliative radiation alone, and the study did not have access to previous treatment history for two patients.

The patients had a median follow-up of 11 months (range, 0.1-146 months) after which the survival analysis was performed. Out of these 207 patients, 19 (9.2\%) were found to have metastatic lesions in the skeletal system. This included 12 out of 19 patients (63.2\%) who were males. Patients received a median number of 2 treatment lines (range, $0-4$ ). The primary tumor was located in the pancreatic body/tail in 12 out of the 19 patients $(63.2 \%)$ who had SM. The disease was locally advanced in 4 out of 19 patients $(21 \%)$ with $79 \%$ being metastatic at the time of initial diagnosis. The number of metastatic lesions at different sites of SM in decreasing order of incidence were thoracic vertebrae in 10 patients, lumbar vertebrae in 6 patients, ribs in 6 patients, pelvis in 5 patients, sacrum in 4 patients, scapula in 3 patients, acetabulum in 2 patients, cervical vertebrae in 2 patients, femoral head in 2 patients, sternum in 1 patient and humeral head in 1 patient. Figure 1 shows a CT scan demonstrating both osteoblastic and osteolytic skeletal mestastases. Other common sites of synchronous or metachronous visceral metastatic lesions in these patients with SM were the liver in 13 patients, lung in 7 patients, peritoneum in 2 patients, non-regional lymph nodes in 2 patients, spleen in 1 patient, pericardium in 1 patient, and abdominal wall in 1 patient. Table 1 outlines the patients' demographic and clinical characteristics of the whole cohort as well as the patients who developed SM. 
Table 1 Characteristics of the whole cohort and patients with skeletal metastases (SM)

\begin{tabular}{|c|c|c|}
\hline Characteristics & $\begin{array}{l}\text { Patients with skeletal } \\
\text { metastases }(n=19)\end{array}$ & Whole cohort $(n=207)$ \\
\hline Age (years), median [range] & $65.5[53-91]$ & 66 [53-92] \\
\hline Primary tumor in pancreatic body/tail, n (\%) & $12(63.2)$ & $78(37.7)$ \\
\hline Most common other metastatic sites & Liver (68.4\%), lung (36.8\%) & Liver (24.6\%), lung (7.7\%), peritoneum (7.2\%) \\
\hline Median number of lines of treatment, median [range] & $2[0-4]$ & 1 [0-6] (2 patients with unknown treatment history) \\
\hline
\end{tabular}

Table 2 Characteristics of patients with skeletal metastases (SM)

\begin{tabular}{ll}
\hline Characteristics & Value (N=19) \\
\hline Most common site of metastases, [n] & Thoracic vertebrae [10], lumbar vertebrae [6] \\
Number of other metastatic sites, median [range] & $1[0-4]$ \\
Most common other metastatic sites, [n] & Liver [13], lung [7] \\
Osteolytic lesions, $\mathrm{n}(\%)$ & $12(63.2)$ \\
Bone pain as initial symptom, $\mathrm{n}(\%)$ & $7(36.8)$ \\
Pathological fracture, $\mathrm{n}(\%)$ & $2(10.5)$ \\
Para-spinal mass causing inferior vena cava obstruction, $\mathrm{n}(\%)$ & $1(5.3)$ \\
Median time of diagnosis of SM from initial diagnosis (months), median [range] & 2 [0-60] \\
Treatment of SM, [n] & Radiation [5], denosumab [3] \\
\hline
\end{tabular}

Most of the skeletal lesions were osteolytic (12 out of 19 , or $63.2 \%$ ) with a median time of diagnosis of SM from initial diagnosis being 2 months (range, 0-60 months). SM were present at the time of diagnosis in 8 out of 19 patients (42.1\%) whereas in 11 out of 19 patients (57.9\%) they developed during the course of the treatment. Out of these 19 patients with SM, 13 were discovered by CT (68.4\%), 4 were discovered by FDG-PET $(21.1 \%)$ and 2 were discovered by MRI (10.5\%). SM were identified incidentally when imaging was done for re-staging and further treatment planning in 11 patients $(57.9 \%)$, while 3 patients were diagnosed on imaging after presenting with SM related pain (15.8\%) and 5 patients were diagnosed after imaging was performed for nonspecific complaints like abdominal pain or dyspnea $(26.3 \%)$. Bone pain was observed as the initial symptom in 7 out of 19 patients (36.8\%). Other complications were seen in 3 patients, where 2 out of $19(10.5 \%)$ had a pathological fracture and 1 out of $19(5.3 \%)$ had a para-spinal mass secondary to a skeletal metastatic lesion which was causing inferior vena cava (IVC) compression. Out of the 19 patients, 8 received treatment for the skeletal lesions (42.1\%)—denosumab was used in 3 patients and palliative radiotherapy was given to the other 5 patients. Table 2 summarizes clinical characteristics of patients with SM.

Out of the 19 patients with SM, 1 patient (5.3\%) had an ECOG PS of 0, 12 patients (63.2\%) had an ECOG PS of 1, 4 patients (21.1\%) had an ECOG PS of 2 and 2 patients (10.5\%) had an ECOG PS of 3. A poor ECOG PS was not statistically correlated with the incidence of $\mathrm{SM}(\mathrm{P}=0.48)$, as shown in Table 3. The median overall survival (mOS) for patients with SM was 11 months (range, 0-62 months). The median survival from onset of SM was 4 months (range, 0-14 months), shown in Table 4. The mOS of patients without SM was 12 months (range, 0-147 months). Survival analysis by a Kaplan-Meier curve and fitted cox 
Table 3 Correlation of ECOG performance status with skeletal metastases

\begin{tabular}{|c|c|c|c|c|c|}
\hline Variables & $\mathrm{ECOG}=0$ & $\mathrm{ECOG}=1$ & $\mathrm{ECOG}=2$ & $\mathrm{ECOG}=3$ & $P$ value \\
\hline Number of patients with skeletal metastases $(\mathrm{N}=19), \mathrm{n}(\%)$ & $1(5.3)$ & $12(63.2)$ & $4(21.1)$ & $2(10.5)$ & \\
\hline
\end{tabular}

ECOG, Eastern Cooperative Oncology Group; PS, performance status.

Table 4 Overall survival in sub-groups of patients with skeletal metastases

\begin{tabular}{lc}
\hline Skeletal metastases status & Median overall survival (months) [range] \\
\hline Patients with skeletal metastases & $11[0-62]$ \\
Patients without skeletal metastases & $12[0-147]$ \\
Survival from onset of skeletal metastases & $4[0-14]$ \\
\hline
\end{tabular}

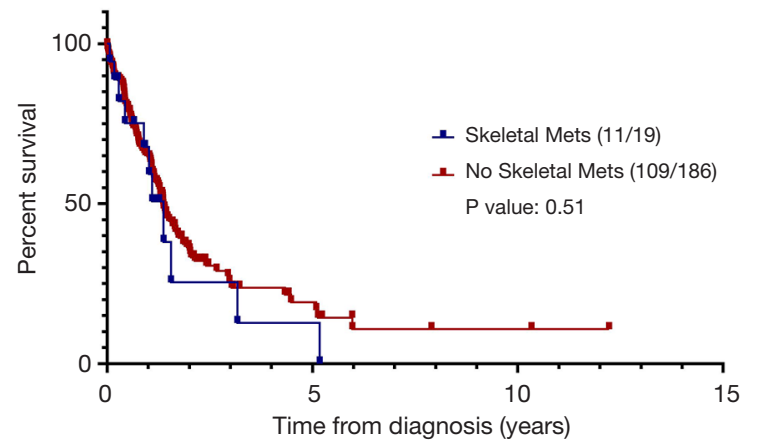

Figure 2 Estimated OS (years) in patients with and without skeletal metastases. OS, overall survival; Mets, metastases.

proportional hazards ratio did not show a statistically significant difference between the overall survival in patients with and without SM [hazard ratio (HR) 1.24, $95 \%$ confidence interval (CI): 0.66-2.30, $\mathrm{P}=0.51$ ) (Figure 2 and Table 5).

\section{Discussion}

SM pose a considerable risk of complications such as immobilization, loss of independence, and reduced quality of life in patients with metastatic malignancies (12). In addition, pain from the bone metastases is a cause of impaired PS and psychological distress among cancer patients (18). These might also result in further sequelae such as pathological fractures, hypercalcemia and organ compression. This is particularly complicated by the fact that symptomatic presentation might be mistakenly thought to be a result of the underlying primary malignancy (e.g., back pain) or might be accompanied by other non-specific complaints. Early diagnosis of SM in such patients is of paramount importance towards reducing morbidity through early and effective interventions (19).

As per the data in our study, the incidence of SM in patients with advanced PDAC has increased from the incidence previously reported in literature of less than $2 \%$ to $9.1 \%$ in this study. A majority of the patients $(68.4 \%)$ in this study had an ECOG PS of 0 or 1 . Thus, the occurrence of SM did not seem to correlate with a poor PS. The primary tumor location being in the body/tail of the pancreas in 12 out of the 19 patients $(63.2 \%)$ and the most common sites of SM being the thoracic vertebrae followed by the lumbar vertebrae and pelvis were in concurrence with the reports in the literature. The predominant nature of SM lesions associated with PDAC being of osteolytic or osteoblastic nature has been variable in different studies $(9,20)$. The data from this study shows that $63.2 \%$ of the SM lesions were osteolytic. Out of the 19 patients with SM, 8 patients $(42.1 \%)$ were found to have SM at the time of diagnosis, whereas 11 patients $(57.9 \%)$ were found to develop SM at some point during the course of their disease and treatment. The time of SM diagnosis from the initial diagnosis of cancer also varied from 0 to 60 months. The SM resulted in considerate morbidity as symptomatic disease was present in 7 out of the 19 patients (36.8\%) with 2 experiencing complications of a pathological fracture and 1 patient who was found to have IVC compression from a SM-related para-spinal mass. 
Table 5 Fitted cox proportional hazards ratio for OS by skeletal metastases status

\begin{tabular}{lccc}
\hline Group & Hazard ratio & $95 \% \mathrm{Cl}$ & $\mathrm{P}$ value \\
\hline Skeletal metastases vs. no skeletal metastases & 1.24 & $0.66-2.30$ & 0.51 \\
\hline
\end{tabular}

OS, overall survival; $\mathrm{Cl}$, confidence interval.

There has been a lack of survival analysis in the literature in patients with and without SM in advanced PDAC. In this study, the outcomes comparing the advanced pancreatic cancer patients with and without SM were analyzed; however, there was no statistically significant OS difference between the two groups, indicating that the patients having SM might not have poorer outcomes than those without them.

FDG-PET and CT appear to have a complementary role in the detection of distant metastases in patients with pancreatic cancer. As per a study of 16 patients with metastatic pancreatic adenocarcinoma, FDG-PET correctly identified 8 metastatic sites in 7 patients, including bone metastases which were missed on CT imaging. Based on the results of whole body FDG-PET, the clinical stage was changed in 5 out of 42 patients (11.9\%) (21). As per this study, no imaging modality appears to have a superior detection rate; however, when used in conjunction, the rates of detection may be much higher (21). In this study, a majority of the SM were diagnosed with a CT scan (13 out of 19 patients, $68.4 \%$ ). The intent of most of these CTs was to stage or re-stage the PDAC and to decide on further treatment strategy (11 out of 19 patients with SM, 57.9\%). Only a few patients were symptomatic at the time of diagnosis, with 3 patients having SM related bone pain and 5 patients having nonspecific complaints that led to imaging and diagnosis of SM. Furthermore, it is also thought that serial measurement of C-telopeptide could enhance early detection of asymptomatic SM, but further validation is needed before we could use this as a screening or diagnostic tool (13).

The benefit of therapies for SM such as bisphosphonates, denosumab and palliative radiation should be evaluated on a case-by-case basis. These would likely benefit those patients who have symptoms from SM. They may also benefit patients with better PS, locally advanced $v s$. metastatic disease at the time of diagnosis of SM, and may possibly contribute to prolonged overall survival.

An important limitation of this study is the existing medical surveillance bias. Only patients with available follow-up information regarding the study variables were included in the study. Thus, there is a proportion of patients who were possibly lost due to lack of follow-up, refused imaging, etc. who were not included in the study and could impact the incidence rate of $S M$ in the patient population. Since the data was collected retrospectively, the reporting of patient-related complaints had minimal interviewer bias.

As per the results of this study, it is evident that the incidence of SM, both symptomatic and asymptomatic, in patients with advanced PDAC is increased from the previously established $2 \%$ in literature (12). There is no published evidence studying survival outcomes of these patients; as per this study, we found no overall survival difference but SM still pose a risk of increased morbidity due to the related complications. Thus, it raises an important question of devising a planned imaging strategy for SM screening in patients with advanced PDAC. This will facilitate early detection of SM and their management to decrease the complications and morbidity associated with them. This study forms the ground work for future studies which can be done at a multi-institutional platform, including a larger number of patients and provide corroborative evidence to these findings. There is also a need to address the question of needing dedicated skeletal imaging such as bone scan or PET scan in a subset of advanced pancreatic cancer patients who seem to be at higher risk to develop SM. As per this study, the increase in the incidence of SM is small but was seen predominantly in males, patients with primary tumor in pancreatic body/ tail, those diagnosed with metastatic cancer at initial presentation with liver and lung metastatic lesions, and those who have received more than one line of therapy. The range of patients included in this study was broadmen and women from 53 to 92 years were included, and most of the patients received standard of care treatments. The results of the study can be applied to other settings of pancreatic cancer patients, as we included a cohort of patients which is a good representation of the general pancreatic cancer population in terms of age, sex, severity of disease and treatments. Impact of other factors like ethnicity, comorbidities, and genetic differences in patients from other countries need to be studied. 


\section{Conclusions}

The incidence of SM in this study was $9.1 \%$, which is an increase from the less than $2 \%$ reported in prior literature. Thoracic and lumbar vertebrae are the most common sites and pathological fractures in these sites can be catastrophic. The data did not demonstrate a difference in survival in patients with and without SM. However, careful clinical evaluation of skeletal signs and symptoms and radiological evaluation for early detection and intervention are imperative to prevent morbidity and mortality from potential catastrophic complications related to SM. This study establishes the groundwork for future, larger sized studies to analyze the role of dedicated skeletal imaging in a subset of advanced pancreatic cancer patients. There is a need to further study these risk factors such as male gender, primary tumor in pancreatic body/tail, metastatic cancer at initial presentation, the presence of liver and lung metastatic lesions, and those who have received more than one line of therapy to identify which patients may be at a higher risk to develop SM and its related complications.

\section{Acknowledgments}

The author would like to thank Michael Killingsworth for proof reading the manuscript.

Funding: None.

\section{Footnote}

Reporting Checklist: The authors have completed the STROBE reporting checklist Available at http://dx.doi. org/10.21037/jgo-20-361

Data Sharing Statement: Available at http://dx.doi. org/10.21037/jgo-20-361

Conflicts of Interest: All authors have completed the ICMJE uniform disclosure form (available at http://dx.doi. org/10.21037/jgo-20-361). Dr. BN reports other from Advanced Accelerator Applications, a Novartis Company, personal fees from Advanced Accelerator Applications, a Novartis Company, personal fees from Lexicon Pharmaceuticals, Inc., personal fees from Novocure Inc., personal fees from Ipsen Biopharmaceuticals, Inc., other from Lexicon Pharmaceuticals, Inc., personal fees from Sun Pharmaceutical, outside the submitted work. Dr. MK reports that he is a consultant for Bayer and Amgen and received research funding from Celgene and Chronix. The other authors have no conflicts of interest to declare.

Ethical Statement: The authors are accountable for all aspects of the work in ensuring that questions related to the accuracy or integrity of any part of the work are appropriately investigated and resolved. The study was conducted in accordance with the Declaration of Helsinki (as revised in 2013). The study was approved by the Institutional Review Board at Banner MD Anderson Cancer Center. (Project \# 14-15-0094) and individual consent for this retrospective analysis was waived.

Open Access Statement: This is an Open Access article distributed in accordance with the Creative Commons Attribution-NonCommercial-NoDerivs 4.0 International License (CC BY-NC-ND 4.0), which permits the noncommercial replication and distribution of the article with the strict proviso that no changes or edits are made and the original work is properly cited (including links to both the formal publication through the relevant DOI and the license). See: https://creativecommons.org/licenses/by-nc-nd/4.0/.

\section{References}

1. Poruk KE, Firpo MA, Adler DG, et al. Screening for pancreatic cancer: why, how, and who? Ann Surg 2013;257:17-26.

2. Distler M, Ruckert F, Hunger M, et al. Evaluation of survival in patients after pancreatic head resection for ductal adenocarcinoma. BMC Surg 2013;13:12.

3. Burris HA 3rd, Moore MJ, Andersen J, et al. Improvements in survival and clinical benefit with gemcitabine as firstline therapy for patients with advanced pancreas cancer: a randomized trial. J Clin Oncol 1997;15:2403-13.

4. Kundranda M, Dragovich T. Nab-paclitaxel plus gemcitabine in the treatment of metastatic pancreatic cancer: utility and experience from the clinic. Gastrointest Cancer 2016;6:13-20.

5. Moore MJ, Goldstein D, Hamm J, et al. Erlotinib plus gemcitabine compared with gemcitabine alone in patients with advanced pancreatic cancer: a phase III trial of the National Cancer Institute of Canada Clinical Trials Group. J Clin Oncol 2007;25:1960-6.

6. Conroy T, Desseigne F, Ychou M, et al. FOLFIRINOX versus gemcitabine for metastatic pancreatic cancer. N Engl J Med 2011;364:1817-25.

7. Von Hoff DD, Ervin T, Arena FP, et al. Increased survival 
in pancreatic cancer with nab-paclitaxel plus gemcitabine. N Engl J Med 2013;369:1691-703.

8. Kundranda M, Kachaamy T. Promising new therapies in advanced pancreatic adenocarcinomas. Future Oncol 2014;10:2629-41.

9. Joffe N, Antonioli D. Osteoblastic bone metastases secondary to adenocarcinoma of the pancreas. Clin Radiol 1978;29:41-6.

10. Lipko AA. On some features of the course of lung and bone metastases of pancreatic cancer. Vopr Onkol 1963;9:80-5.

11. Pneumaticos SG, Savidou C, Korres DS, et al. Pancreatic cancer's initial presentation: back pain due to osteoblastic bone metastasis. Eur J Cancer Care (Engl) 2010;19:137-40.

12. Borad MJ, Saadati H, Lakshmipathy A, et al. Skeletal metastases in pancreatic cancer: a retrospective study and review of the literature. Yale J Biol Med 2009;82:1-6.

13. Iguchi H, Yasuda M, Matsuo T, et al. Nihon Shokakibyo Gakkai Zasshi 2004;101:872-8.

14. Käkönen SM, Mundy GR. Mechanisms of osteolytic bone metastases in breast carcinoma. Cancer 2003;97:834-9.

Cite this article as: Puri A, Chang J, Tanner N, Lucente P, Desiongco J, Dragovich T, Naraev B, Kundranda M. Skeletal metastases in advanced pancreatic ductal adenocarcinoma: a retrospective analysis. J Gastrointest Oncol 2021;12(2):455-463. doi: 10.21037/jgo-20-361
15. Peavy PW, Rogers JV Jr, Clements JL Jr, et al. Unusual osteoblastic metastases from carcinoid tumors. Radiology 1973;107:327-30.

16. Saif MW, Galanina N, Ravage-Mass L, et al. Bone Metastasis as the Only Metastatic Site in a Patient with Pancreatic Cancer following Distal Pancreatectomy. Case Rep Med 2010;2010:634975.

17. Ohira Y, Kaga K, Kodama A. Nihon Jibiinkoka Gakkai Kaiho 1991;94:9-15.

18. Clohisy DR, Mantyh PW. Bone cancer pain. Clin Orthop Relat Res 2003;(415 Suppl):S279-88.

19. Clézardin P, Ebetino FH, Fournier PG. Bisphosphonates and cancer-induced bone disease: beyond their antiresorptive activity. Cancer Res 2005;65:4971-4.

20. Mao C, Domenico D, Kim K, et al. Observations on the developmental patterns and the consequences of pancreatic exocrine adenocarcinoma: Findings of 154 autopsies. Arch Surg 1995;130:125-34.

21. Nishiyama Y, Yamamoto Y, Yokoe K, et al. Contribution of whole body FDG-PET to the detection of distant metastases in pancreatic cancer. Ann Nucl Med 2005;19:491-7. 